\title{
PREDICTION OF SIGNAL ATTENUATION DUE TO DUSTSTORMS USING MIE SCATTERING
}

\author{
Md. RAFiQul ISLAM, ZAin Elabdin, OMER ElshaikH, OTHMAN O. KHALIFA, \\ AHM Zahirul Alam, Sheroz Khan AND A. W. NAJI \\ Wireless Communication and Signal Processing Research Group, \\ Kulliyyah of Engineering, International Islamic University Malaysia \\ P.O. Box 10, Kuala Lumpur, 50728, Malaysia. \\ E-mail: rafiq@iiu.edu.my
}

\begin{abstract}
The present trend in radio design calls for the use of frequencies above 40 $\mathrm{GHz}$ for short links carrying wide-band digital communication signals. In order to utilize the new frequency band efficiently, signal attenuation studies due to duststorms is needed urgently for desert areas. This paper presents a mathematical model which has been developed to predict the signal attenuation due to duststorm. The proposed model enables the convenient calculation of the signal path attenuation based on Mie solution of Maxwell's equations for the scattering of electromagnetic wave by dust particles. The predicted values from the proposed mathematical model are compared with the measured values observed in Saudi Arabia and Sudan and show relatively close agreement.
\end{abstract}

Keywords: Signal Attenuation, Duststorm, Mathematical Model, Duststorm Visibility.

\section{ABSORPTION AND SCATTERING BY THE ATMOSPHERE}

When microwaves pass through the medium containing precipitations like rain, snow or dust particles, the signals get attenuated through two phenomena [1].

a) Absorption of energy by these particles.

b) Scattering of energy out of the beam by these particles.

Microwaves suffer absorption and scattering by the atmosphere especially at higher frequencies where the scattering effects become more severe [2]. The knowledge of these characteristics is essential to design reliable communications.

\subsection{Theory of single-particle scattering}

The basic theory underling mathematical model for attenuation is the theory for singleparticle scattering [3]. The characteristics of these two phenomena, scattering and absorption, can be expressed most conveniently by assuming an incident plane wave. The total electromagnetic field around the scattering object is split into an incident and a scattered field as shown in Fig. 1 [4]: 


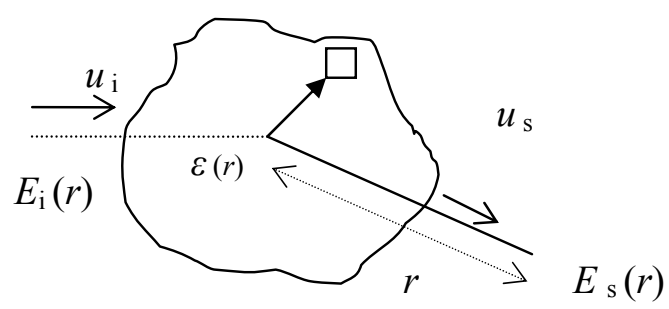

Fig. 1: Scattering configuration.

The total amplitude of the fields at any point on the surface of the sphere can be given as [5]:

$$
\begin{gathered}
H=H_{i}+H_{s} \\
E=E_{i}+E_{s}
\end{gathered}
$$

where, $E_{i}$ and $H_{i}$ denote the incident electric and magnet field, and $E_{s}$ and $H_{s}$ denote corresponding scattered fields.

\subsection{The total cross-section efficiency factors}

The power density of the incident wave is uniform in space. Therefore, the power loss of the incident wave can be calculated by multiplying the uniform power density with a cross-section, having the dimension of the area. The total power removed from the incident field by absorption and scattering can be represented by the total or extinction cross-section [4].

When the problem is concerned with the flow of energy, poynting vector must be used. So the power density is represented by the averaged poynting vector $\bar{S}$. This poynting vector can be splitted into separate terms using the separation of the total field into the incident and scattered field (Eq. (1) and Eq. (2)), resulting in [4]:

$$
\bar{S}=\bar{S}_{i}+\bar{S}_{s}+\bar{S}_{d}
$$

where,

$$
\begin{aligned}
& \overline{S_{i}}=1 / 2 \operatorname{Re}\left(E_{i} \times H_{i}{ }^{*}\right) \\
& \overline{S_{s}}=1 / 2 \operatorname{Re}\left(E_{s} \times H_{s}^{*}\right) \\
& \bar{S}_{d}=1 / 2 \operatorname{Re}\left(E_{i} \times H_{s} *+E_{s} \times H_{i}^{*}\right)
\end{aligned}
$$


and where, $E_{i}$ and $H_{i}$ denote the incident electric and magnet field, whereas $E_{s}$ and $H_{s}$ denote corresponding scattered fields.

The net power flow through the surface of this sphere can be calculated by integrating the radial component of $\bar{S}$ over the sphere. If the scatterer is a lossless dielectric, the power flow will be zero. If it is lossy (permittivity is complex), the net power flow will represent the absorption rate $P_{a}$ of energy by the scatterer as shown in Fig. 2 and can be written as:

$$
-P_{a}=P_{i}+P_{s}+P_{d}
$$

where $P_{a}$ represents the integral of the radial component of $\bar{S}$.

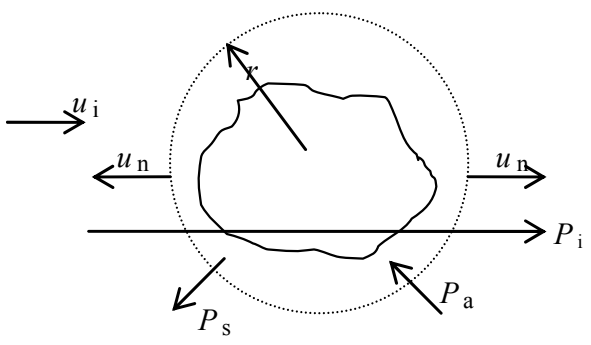

Fig. 2: Scattering points.

The medium surrounding the particle is assumed to be lossless, so the net flow of the energy of the incident field is zero for every closed surface $\left(P_{\mathrm{i}}=0\right)$, and thus:

$$
P_{a}+P_{s}=-P_{d}
$$

and from Eq. 6;

$$
P_{a}+P_{s}=-P_{d}=-1 / 2 \operatorname{Re} \iint_{s}\left(E_{i} \times H_{s}^{*}+E_{s} \times H_{i}^{*}\right) \cdot u_{n} d S
$$

where $u_{n}$ is the unit vector normal to the surface $S$ and directed outwards and the dot represent the inner product as shown in Fig. 2.

Using Maxwell equation the sum of absorbed and scattered energy can be expressed as [4]:

$$
P_{a}+P_{s}=\frac{2 \pi}{k}\left(\frac{\varepsilon}{\mu}\right)^{1 / 2} E_{i} \operatorname{Im}\left[u_{e} \cdot f\left(u_{s}, u_{i}\right)\right]
$$

where,

$k \quad$ : wave number $(k=\omega \sqrt{\mu \varepsilon}=(2 \pi) / \lambda)$. 
$\left(\frac{\varepsilon}{\mu}\right)^{1 / 2}:$ characteristic impedance of the medium.

$u_{\mathrm{e}} \quad$ : unit vector defining the polarization of the wave.

$f\left(u_{s}, u_{i}\right)$ : the scattering amplitude function; it is a function of the direction of propagation $u_{i}$ of the incident field and of the direction $u_{\mathrm{s}}$ from the object to the observation point.

The ratio of the rate of removal of energy $\left(P_{a}+P_{s}\right)$ to the rate of the incident energy $\left(P_{i}\right)$ on a unit cross-sectional area of the scatterer is defined as the extinction or total crosssection efficiency factors [4]:

$$
\sigma_{t}=\frac{P_{a}+P_{s}}{P_{i}}=\sigma_{a}+\sigma_{s}=\frac{4 \pi}{k} \operatorname{Im}\left[u_{e} \cdot f\left(u_{s}, u_{i}\right)\right]
$$

\section{ANALYTICAL MODELS FOR SCATTERING}

Two models have been presented for an analytical solution of Maxwell's equations for the scattering of electromagnetic wave by dielectric spherical particles, Rayleigh approximation and Mie solution.

\subsection{Rayleigh Approximation}

The assumption for Rayleigh approximation is that $(k a<1)$, meaning that the radius a of the particle is small compared with the wavelength. For a dielectric sphere that is small compared with wavelength, the field inside the particle is chosen to be the solution of the equivalent dielectric electrostatic problem. In the Rayleigh approximation, the field inside the scattering particle is not modeled properly. For an exact solution the scattered field reflects all the properties of the object which is no longer true for an approximation solution [5].

The extinction or total cross-section efficiency factors by Rayleigh approximation expressed as [6]:

$$
\sigma_{t}=\sigma_{s}+\sigma_{a}=\left[\frac{8}{3} \pi a^{2}(k a)^{4}+12 \pi a^{2}(k a) \frac{\varepsilon^{\prime \prime}}{\left(\varepsilon^{\prime}-1\right)^{2}+\left(\varepsilon^{\prime \prime}\right)^{2}}\right]\left|\frac{\varepsilon-1}{\varepsilon+2}\right|^{2}
$$

where,

$a:$ the particle radius

$k:$ the wavenumber $(k=2 \pi / \lambda)$

$\varepsilon^{\prime}, \varepsilon^{\prime \prime}:$ the real and imaginary contributions of the relative dielectric constant of the particles 


\subsection{Mie Solution}

Mie solution is a complete analytical solution of Maxwell's equations for the scattering of electromagnetic wave by dielectric spherical particles. In contrast to Rayleigh scattering Mie solutions to scattering embraces all possible ratios of diameter to wavelength [7].

Mie solutions unlike Rayleigh scattering do not depend upon any such limitation and can be utilized to predict attenuation in microwave wave band with high reliability especially at higher frequencies used by new systems with larger bandwidth.

The extinction or total cross-section efficiency factors by Mie solutions can be expressed as [6]:

$$
\sigma_{t}=\frac{\lambda^{2}}{2 \pi}(k a)^{3}\left(c_{1}+c_{2}(k a)^{2}+c_{3}(k a)^{3}\right)
$$

where,

$a:$ the particle radius

$k:$ the wavenumber $(k=2 \pi / \lambda)$

$\lambda:$ the wavelength, and

$C_{1}, C_{2}$ and $C_{3}$ : constants whose values depend on real $\left(\varepsilon^{\prime}\right)$ and imaginary part $\left(\varepsilon^{\prime \prime}\right)$ of the dielectric constant of the particles as:

$$
\begin{aligned}
& C_{1}=\frac{6 \varepsilon^{\prime \prime}}{\left(\varepsilon^{\prime}+2\right)^{2}+\varepsilon^{\prime \prime 2}} \\
& C_{2}=\varepsilon^{\prime \prime}\left\{\frac{6}{5} \frac{\varepsilon^{\prime 2}+7 \varepsilon^{\prime \prime 2}+4 \varepsilon^{\prime}-20}{\left[\left(\varepsilon^{\prime}+2\right)^{2}+\varepsilon^{\prime \prime 2}\right]^{2}}+\frac{1}{15}+\frac{5}{3\left[\left(2 \varepsilon^{\prime}+3\right)^{2}+4 \varepsilon^{\prime \prime 2}\right]}\right\} \\
& C_{3}=\frac{4}{3}\left\{\frac{\left(\varepsilon^{\prime}-1\right)^{2}\left(\varepsilon^{\prime}+2\right)+\left[2\left(\varepsilon^{\prime}-1\right)\left(\varepsilon^{\prime}+2\right)-9\right]+\varepsilon^{\prime \prime 4}}{\left[\left(\varepsilon^{\prime}+2\right)^{2}+\varepsilon^{\prime \prime 2}\right]^{2}}\right\}
\end{aligned}
$$

\section{SIGNAL ATTENUATION DUE TO DUSTSTORM}

The theory of attenuation due to sand and duststorm can be explained in terms of scattering and absorption cross section of a single particle. The methods to predict the signal attenuation due to rain effects can be applied for duststorm because the general model for scattering in sand and dust particle populations is essentially the same as that for a population of hydrometeors; both of them are discrete random medium [3]. 
The signal attenuation due to duststorm is estimated generally by solving the forward scattering amplitude function of a single particle $[8,9,10]$. The solution may be carried out using the Rayleigh approximation or Mie solutions. The method depends largely on the wave number and particle radius.

The attenuation of electromagnetic radiation $\left(A_{T}\right)$ over a path of extent $L$ through precipitating particles may be written as [11]:

$$
A_{T}=\int_{0}^{L} A_{p} d x \quad[\mathrm{~dB}]
$$

where $A_{p}(\mathrm{~dB} / \mathrm{km})$ is the specific attenuation characterizing the precipitating particles.

The following expression has been used to calculate the attenuation due to rain $[11,12,13]$ :

$$
A_{p}=4.343 \times 10^{3} \int_{a_{\min }}^{a_{\max }} \sigma_{t}(a) \cdot N(a) d a \quad[\mathrm{~dB} / \mathrm{km}]
$$

where,

$N(a) d a$ : the particles number per unit volume of air with particles radius between $a$ and $a+d a$,

$\sigma_{t} \quad$ : the total attenuation cross section efficiency factors of particle of radius $a$.

\subsection{Duststorm Predicted Model}

Starting from the above, we can expressed the attenuation of electromagnetic radiation $\left(A_{T}\right)$ over a path of extent $L$ through duststorm as:

$$
A_{T}=\int_{0}^{L} A_{d} d x[\mathrm{~dB}]
$$

where $A_{d}$ is the s pecific attenuation characterizing the duststorm which can be expressed as:

$$
A_{d}=4.343 \times 10^{3} \int_{a_{\min }}^{a_{\max }} \sigma_{t}(a) \cdot N(a) d a \quad[\mathrm{~dB} / \mathrm{km}]
$$

and where,

$N(a) d a$ : the dust particles number per unit volume of air with dust particles radius between $a$ and $a+d a$,

$\sigma_{t} \quad$ : the total attenuation cross section efficiency factors of dust particle of radius $a$. 


\section{a. Using Rayleigh Approximation}

Goldhirsh [14], in using the Rayleigh approximation expression for the total crosssection efficiency factor $\left(\sigma_{t}\right)$, expressed the specific attenuation due to duststorm $A_{d}$ as:

$$
A=\frac{2.317 \cdot 10^{-3} \cdot \varepsilon^{\prime \prime}}{\left[\left(\varepsilon^{\prime}+2\right)^{2}+\varepsilon^{\prime \prime 2}\right] \cdot \lambda} \cdot \frac{1}{V^{\gamma}} \quad[\mathrm{dB} / \mathrm{km}]
$$

where,

$V:$ the visibility in kilometer,

$\lambda$ : the wavelength in meter,

$\gamma$ : constants that depend on the type of land from which the storm originated as well as the climatic conditions,

$\varepsilon^{\prime}, \varepsilon^{\prime \prime}$ : the real and imaginary part of the dielectric constant of the dust particles.

Goldhirsh formulation is applicable at wavelengths for which the Rayleigh condition is applicable which is equal to $48 \mathrm{GHz}$ as a maximum frequency [14].

\section{b. Using Mie solution}

In order to derive a mathematical model that can predict the signal attenuation due to duststorm at higher frequencies, the Mie expression for total cross-section efficiency factors $\left(\sigma_{t}\right)$ that appear in Eq. (13) was selected for substitution in Eq. (20). So the duststorm attenuation $\left(A_{d}\right)$ can be written as:

$$
A_{d}=4.343 \times 10^{3} \int_{a_{\min }}^{a_{\max }} \frac{\lambda^{2}}{2 \pi}(k a)^{3}\left(c_{1}+c_{2}(k a)^{2}+c_{3}(k a)^{3}\right) \cdot N(a) d a \quad[\mathrm{~dB} / \mathrm{km}]
$$

\section{i. Dependence on Visibility}

To calculate the attenuation by Eq. (22) requires data for the number of particles of dust $N$, which is difficult to measure accurately. On the other hand, statistical information on duststorm visibility is available. Goldhirsh [14] expresses the visibility in term of the particle density and the radius as:

$$
V(\mathrm{~km})=\frac{5.5 \times 10^{-4}}{N a_{e}{ }^{2}}
$$

where,

$N$ : the dust particles number per unit volume,

$a_{\mathrm{e}}:$ the equivalent particle radius in meters. 
By solving $N$ in the above formula we can express the particle density in term of the visibility and the radius as:

$$
N=\frac{5.5 \times 10^{-4}}{V a_{e}{ }^{2}}
$$

\section{ii. General Formula}

By substituting the particle density expression which appear in Eq. (24) into Eq. (22), the specific attenuation characterizing the duststorm $\left(A_{d}\right)$ may alternately be expressed as:

$A_{d}=4.343 \times 10^{3} \int_{a_{\min }}^{a_{\max }}\left[\frac{\lambda^{2}}{2 \pi}(k a)^{3}\left(c_{1}+c_{2}(k a)^{2}+c_{3}(k a)^{3}\right) \cdot \frac{5.5 \times 10^{-4}}{V a_{e}{ }^{2}}\right] d a[\mathrm{~dB} / \mathrm{km}]$.

A further approximation can be made in these calculations, assuming that every dust particle in a real storm may be replaced by an equivalent particle $\left(a_{e}\right)$ whose radius is the mean radius for all dust particle. By this assumption the value of equivalent particle radius $\left(a_{e}\right)$ considered as constant value and Eq. (23) may alternately be expressed as an algebraic expression:

$$
A_{d}=4.343 \times 10^{3} \times\left[\frac{\lambda^{2}}{2 \pi}\left(k a_{e}\right)^{3}\left(c_{1}+c_{2}\left(k a_{e}\right)^{2}+c_{3}\left(k a_{e}\right)^{3}\right) \cdot \frac{5.5 \times 10^{-4}}{V a_{e}{ }^{2}}\right][\mathrm{dB} / \mathrm{km}] .
$$

and can be simplified to:

$$
A_{d}=\frac{0.38 \times \lambda^{2}}{V}\left(c_{1} k^{3} a_{e}+c_{2} k^{5} a_{e}^{3}+c_{3} k^{6} a_{e}^{4}\right) \quad[\mathrm{dB} / \mathrm{km}]
$$

By substituting $k=2 \pi / \lambda$ in Eq. (25):

$$
A_{d}=\frac{0.38}{V}\left(c_{1} \frac{8 \pi^{3}}{\lambda} a_{e}+c_{2} \frac{32 \pi^{5}}{\lambda^{3}} a_{e}^{3}+c_{3} \frac{64 \pi^{6}}{\lambda^{4}} a_{e}^{4}\right)[\mathrm{dB} / \mathrm{km}] .
$$

which can be simplified to:

$$
A_{d}=94.3 \times c_{1} \frac{a_{e}}{V \lambda}+3721.2 \times c_{2} \frac{a_{e}^{3}}{V \lambda^{3}}+23381 \times c_{3} \frac{a_{e}{ }^{4}}{V \lambda^{4}} \quad[\mathrm{~dB} / \mathrm{km}] .
$$

Finally, we can express the specific attenuation due to duststorm $A_{d}(\mathrm{~dB} / \mathrm{km})$ as:

$$
A_{d}=\frac{X a_{e}}{V \lambda}+\frac{Y a_{e}^{3}}{V \lambda^{3}}+\frac{Z a_{e}{ }^{4}}{V \lambda^{4}} \quad[\mathrm{~dB} / \mathrm{km}] .
$$

where,

$$
a_{\mathrm{e}}: \text { the equivalent particle radius in meters, }
$$


$V:$ the visibility in kilometer,

$\lambda:$ the wavelength in meter and;

$X, Y$ and $Z$ : constants whose values depend on real $\left(\varepsilon^{\prime}\right)$ and imaginary part $\left(\varepsilon^{\prime \prime}\right)$ of the dielectric constant of the dust particles as:

$$
\begin{gathered}
X=\frac{565.8 \varepsilon^{\prime \prime}}{\left(\varepsilon^{\prime}+2\right)^{2}+\varepsilon^{\prime \prime 2}} \\
Y=3.7 \times 10^{3} \cdot \varepsilon^{\prime \prime}\left\{\frac{6}{5} \frac{7 \varepsilon^{\prime 2}+7 \varepsilon^{\prime \prime 2}+4 \varepsilon^{\prime}-20}{\left[\left(\varepsilon^{\prime}+2\right)^{2}+\varepsilon^{\prime \prime 2}\right]^{2}}+\frac{1}{15}+\frac{5}{3\left[\left(2 \varepsilon^{\prime}+3\right)^{2}+4 \varepsilon^{\prime \prime 2}\right]}\right\} \\
Z=3.12 \times 10^{4}\left\{\frac{\left(\varepsilon^{\prime}-1\right)^{2}\left(\varepsilon^{\prime}+2\right)+\left[2\left(\varepsilon^{\prime}-1\right)\left(\varepsilon^{\prime}+2\right)-9\right]+\varepsilon^{\prime \prime 4}}{\left[\left(\varepsilon^{\prime}+2\right)^{2}+\varepsilon^{\prime \prime 2}\right]^{2}}\right\}
\end{gathered}
$$

\section{iii. The Formula in Term of Frequencies $(f)$ in $\mathbf{G H z}$}

For more simplification of Eq. (31) it is better to express the wavelength $(\lambda)$ in term of frequencies $(f)$ in $\mathrm{GHz}$ which is easier to use by microwave network engineers. So the specific attenuation of duststorm $A_{d}(\mathrm{~dB} / \mathrm{km})$ can be expressed as:

$$
A_{d}=\frac{X a_{e} f}{(0.3) V}+\frac{Y a_{e}^{3} f^{3}}{(0.3)^{3} V}+\frac{Z a_{e}^{4} f^{4}}{(0.3)^{4} V}[\mathrm{~dB} / \mathrm{km}] .
$$

and can be simplified to:

$$
A_{d}=\frac{a_{e} f}{V}\left(x+y a_{e}^{2} f^{2}+z a_{e}^{3} f^{3}\right)[\mathrm{dB} / \mathrm{km}] .
$$

where,

$a_{\mathrm{e}}:$ the equivalent particle radius in meters,

$V:$ the visibility in kilometer,

$f$ : the frequency in $\mathrm{GHz}$, and

$x, y$ and $z$ : constants whose values depend on real $\left(\varepsilon^{\prime}\right)$ and imaginary part $\left(\varepsilon^{\prime \prime}\right)$ of the dielectric constant of the dust particles as: 


$$
\begin{gathered}
x=\frac{1886 \cdot \varepsilon^{\prime \prime}}{\left(\varepsilon^{\prime}+2\right)^{2}+\varepsilon^{\prime \prime 2}} \\
y=137 \times 10^{3} \cdot \varepsilon^{\prime \prime}\left\{\frac{6}{5} \frac{7 \varepsilon^{\prime 2}+7 \varepsilon^{\prime \prime 2}+4 \varepsilon^{\prime}-20}{\left[\left(\varepsilon^{\prime}+2\right)^{2}+\varepsilon^{\prime \prime 2}\right]^{2}}+\frac{1}{15}+\frac{5}{3\left[\left(2 \varepsilon^{\prime}+3\right)^{2}+4 \varepsilon^{\prime \prime 2}\right]}\right\} \\
z=379 \times 10^{4}\left\{\frac{\left(\varepsilon^{\prime}-1\right)^{2}\left(\varepsilon^{\prime}+2\right)+\left[2\left(\varepsilon^{\prime}-1\right)\left(\varepsilon^{\prime}+2\right)-9\right]+\varepsilon^{\prime \prime 4}}{\left[\left(\varepsilon^{\prime}+2\right)^{2}+\varepsilon^{\prime \prime 2}\right]^{2}}\right\}
\end{gathered}
$$

\subsection{Determination of Attenuation at Different Bands}

A further simplification can be made in this general formula (Eq. (33)) to more effective and become easier for use by microwave network designers and engineers. This can be achieved by subdividing the general formula into several formulas, each one can deal with specific microwave band suitable to calculate the signal attenuation due to dust particles at every microwave bands.

It is clear from the general formula that the signal attenuation due to dust particles depends on visibility, frequency, dust particles radius and dielectric constant. The dielectric constant values of dust particles are important components in the determination of attenuation. A number of investigators have measured the refractive index of sand and dust samples [9, 15, 16]. A further approximation can make in the new subdivided formulas, assuming that a single value of the refractive index can be fitted for all frequencies range in specific microwave band.

\section{i. Attenuation at S-band}

For S-band which is in the range between $2-4 \mathrm{GHz}$, the dielectric constants measured were $\varepsilon^{\prime}=4.56, \varepsilon^{\prime \prime}=0.251$ [15]. The specific attenuation of duststorm $A_{d}(\mathrm{~dB} / \mathrm{km})$ can be expressed as:

$$
A_{d S}=11 \cdot \frac{a_{\theta} f}{V}+433 \cdot \frac{a_{e}^{3} f^{3}}{V}+2.46 \times 10^{5} \frac{a_{e}^{4} f^{4}}{V}[\mathrm{~dB} / \mathrm{km}] .
$$

Figure 3 showed the signal attenuation $(\mathrm{dB} / \mathrm{km})$ versus frequency plot at S-bands for four different values of visibility at equivalent particle radius of $50 \mu \mathrm{m}$. 


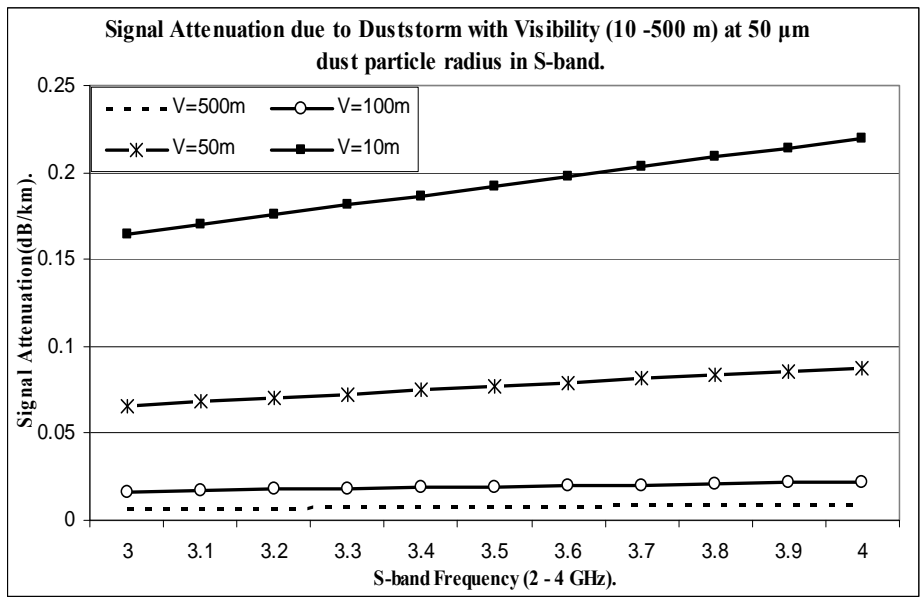

Fig. 3: Signal attenuation $(\mathrm{dB} / \mathrm{km})$ versus frequency at S-band.

\section{ii. Attenuation at X-band}

For X-band which is in the range between $8-12 \mathrm{GHz}$, the dielectric constants measured were $\varepsilon^{\prime}=5.73, \varepsilon^{\prime \prime}=0.415$ [8]. The specific attenuation of duststorm $A_{d}(\mathrm{~dB} / \mathrm{km})$ can be expressed as:

$$
A_{d X}=13 \cdot \frac{a_{e} f}{V}+702 \cdot \frac{a_{e}^{3} f^{3}}{V}+2.5 \times 10^{5} \frac{a_{e}^{4} f^{4}}{V}[\mathrm{~dB} / \mathrm{km}] .
$$

The signal attenuation $(\mathrm{dB} / \mathrm{km})$ versus frequency plot at X-bands for four different values of visibility at equivalent particle radius of $50 \mu \mathrm{m}$ is shown in Fig. 4.

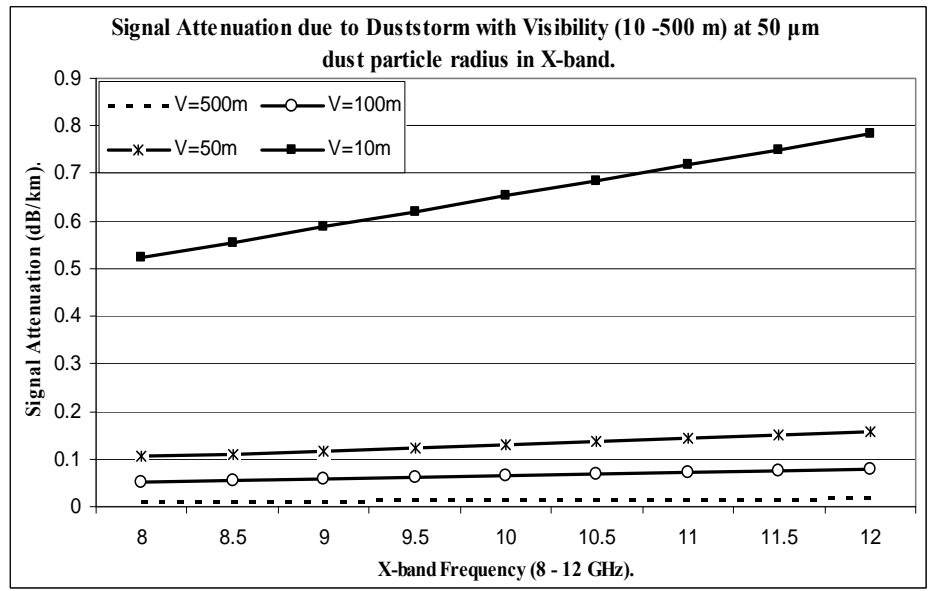

Fig. 4: Signal attenuation $(\mathrm{dB} / \mathrm{km})$ against frequency at X-band. 


\section{iii. Attenuation at Ku-band}

For Ku-band which is in the range between $12-18 \mathrm{GHz}$, the dielectric constants measured were $\varepsilon^{\prime}=5.5, \varepsilon^{\prime \prime}=1.3[16]$. The specific attenuation of duststorm $A_{d}(\mathrm{~dB} / \mathrm{km})$ can be expressed as:

$$
A_{d k u}=42 \cdot \frac{a_{e} f}{V}+2806 \cdot \frac{a_{e}^{3} f^{3}}{V}+2.4 \times 10^{5} \frac{a_{e}^{4} f^{4}}{V} \quad[\mathrm{~dB} / \mathrm{km}]
$$

Figure 5 showed the signal attenuation $(\mathrm{dB} / \mathrm{km})$ versus frequency plot at $\mathrm{Ku}$-bands for four different values of visibility at equivalent particle radius of $50 \mu \mathrm{m}$.

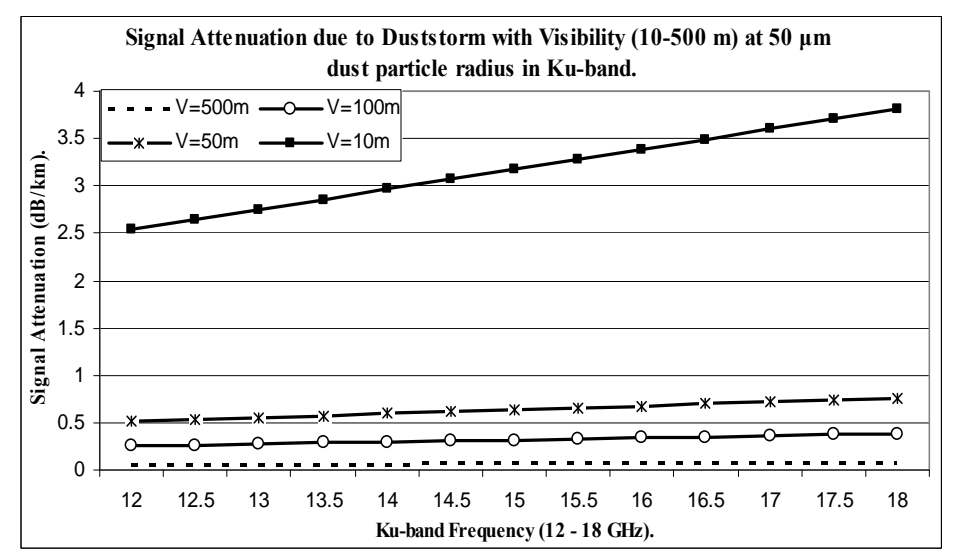

Fig. 5: Signal attenuation $(\mathrm{dB} / \mathrm{km})$ vs frequency at Ku-band.

\section{iv. Attenuation at K-band}

For K-band which in the range between $18-26.5 \mathrm{GHz}$, the dielectric constants measured were $\varepsilon^{\prime}=5.1, \varepsilon^{\prime \prime}=1.4[16]$. The specific attenuation of duststorm $A_{d}(\mathrm{~dB} / \mathrm{km})$ can be expressed as:

$$
A_{d k}=50 \cdot \frac{a_{e} f}{V}+3368 \cdot \frac{a_{e}^{3} f^{3}}{V}+2.38 \times 10^{5} \frac{a_{e}^{4} f^{4}}{V} \quad[\mathrm{~dB} / \mathrm{km}]
$$

The signal attenuation $(\mathrm{dB} / \mathrm{km})$ versus frequency plot at K-bands for four different values of visibility at equivalent particle radius of $50 \mu \mathrm{m}$ is shown in Fig. 6. 


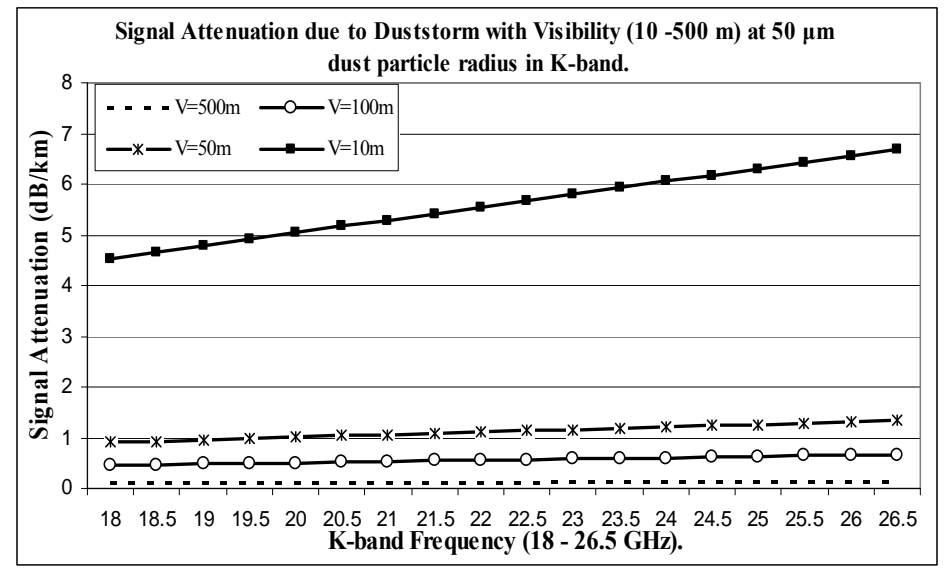

Fig. 6: Signal attenuation $(\mathrm{dB} / \mathrm{km})$ vs frequency at K-band.

\section{v. Attenuation at Ka-band}

For Ka-band which in the range between $26.5-40 \mathrm{GHz}$, the dielectric constants measured were $\varepsilon^{\prime}=4, \varepsilon^{\prime \prime}=1.325$ [16]. The specific attenuation of duststorm $A_{d}(\mathrm{~dB} / \mathrm{km})$ expression can be written as:

$$
A_{d k a}=66 \cdot \frac{a_{e} f}{V}+3712 \cdot \frac{a_{e}^{3} f^{3}}{V}+2.2 \times 10^{5} \frac{a_{e}^{4} f^{4}}{V}[\mathrm{~dB} / \mathrm{km}] .
$$

Figure 7 showed the signal attenuation $(\mathrm{dB} / \mathrm{km})$ versus frequency plot at Ka-bands for four different values of visibility at equivalent particle radius of $50 \mu \mathrm{m}$.

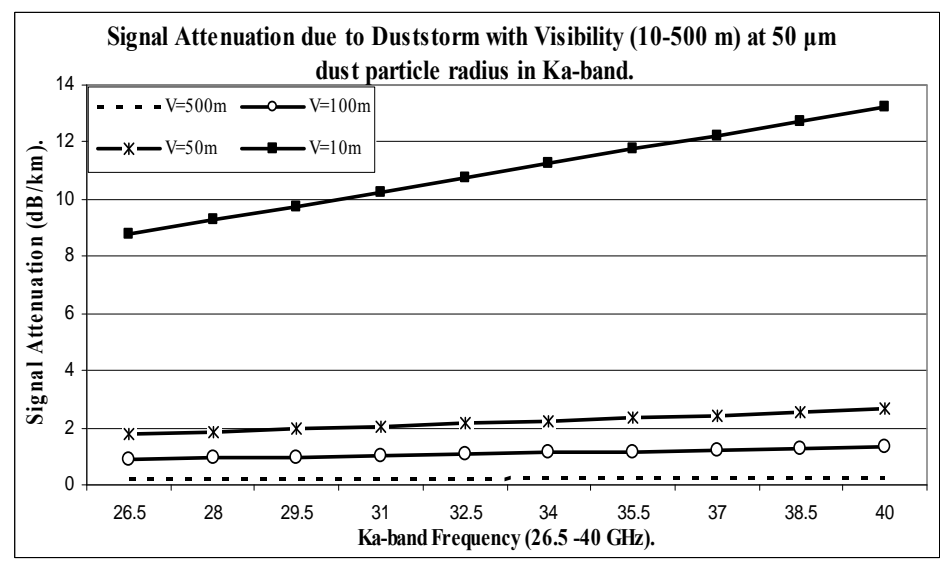

Fig. 7: Signal attenuation $(\mathrm{dB} / \mathrm{km})$ against frequency at Ka-band. 


\section{vi. Attenuation at $\mathbf{W}$-band}

For W-band which in the range between $56-100 \mathrm{GHz}$, the dielectric constants measured were $\varepsilon^{\prime}=3.5, \varepsilon^{\prime \prime}=1.64$ [16]. The specific attenuation of duststorm $A_{d}(\mathrm{~dB} / \mathrm{km})$ expression is;

$$
A_{d W}=94 \cdot \frac{a_{e} f}{V}+6232 \cdot \frac{a_{e}^{3} f^{3}}{V}+2.1 \times 10^{5} \frac{a_{e}^{4} f^{4}}{V}[\mathrm{~dB} / \mathrm{km}] .
$$

The signal attenuation $(\mathrm{dB} / \mathrm{km})$ versus frequency plot at W-bands for four different values of visibility at equivalent particle radius of $50 \mu \mathrm{m}$ is shown in Fig. 8.

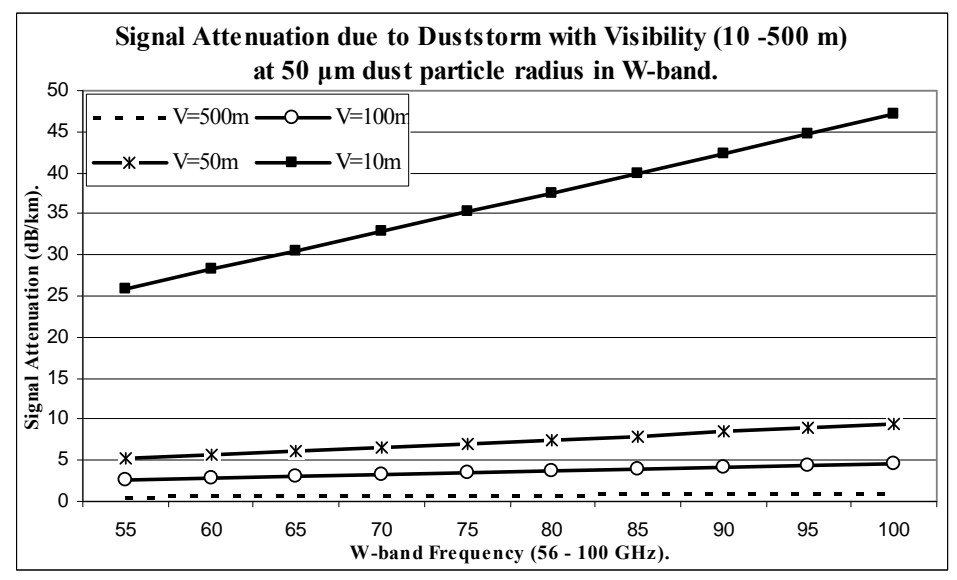

Fig. 8: Signal attenuation $(\mathrm{dB} / \mathrm{km})$ versus frequency at $\mathrm{W}$-band.

\section{VALIDITY OF THE MATHEMATICAL MODEL}

In order to verify the validity of the proposed mathematical model for prediction of signal attenuation due to duststorm, a comparison run between the results obtained from the mathematical model and field measurements were made. At the same time a comparison run between the proposed model and one of the current models was also investigated.

\subsection{Saudi Arabia Measurements}

During the course of measurements, Alhaider and Ali recorded several duststorms during 1987 in the city of Riyadh, Saudi Arabia [17]. The duststorm was observed both by the meteorological sensors and by the radio links. Visibility reduction was measured by using the known marked distance method. For most of the events, the cell size of the storm exceeded $20 \mathrm{~km}$. The measurements run over $14 \mathrm{~km}$ microwave link at $40 \mathrm{GHz}$ (Ka-band). The millimeter wave transmitters and receivers are placed at $100 \mathrm{~m}$ and $25 \mathrm{~m}$ above ground, respectively. 


\subsection{Sudan Measurements}

Measurement in Khartoum-Sudan on September 1, 2007 shows attenuation $0.67 \mathrm{~dB}$ per kilometer observed by the author on $15 \mathrm{~km}$ link at $13 \mathrm{GHz}$ (Ku band) [18]. The duststorm produced a visibility smaller than $5 \mathrm{~m}$. The predicted value found by proposed model using the Ku-band specific attenuation formula is $0.55 \mathrm{~dB}$ per kilometer for the same visibility which is quite close to measured value of $0.67 \mathrm{~dB} / \mathrm{km}$.

\subsection{Comparison of Measured and Calculated Values}

Calculated attenuation values for different values of visibility at $40 \mathrm{GHz}$ are given in Table 1 together with attenuation measured values observed in Saudi Arabia. The predicted values calculated by the proposed Ka-band specific attenuation formula (Eq. 41) and Goldhirsh model (Eq. 21). The following values are considered in calculation:

- Visibility from less than $1 \mathrm{~km}$ to more than $5 \mathrm{~km}$.

- $\quad$ Equivalent dust particle radius $\left(a_{\mathrm{e}}\right)=30 \mu \mathrm{m}$.

- The dielectric constants $\left(\varepsilon^{\prime}=4, \varepsilon^{\prime \prime}=1.325\right)$.

- Frequency $=40 \mathrm{GHz}$.

Table 1: Result of Measurements and Calculations of Signal Attenuation due to Duststorm for Different Value of Visibility at $40 \mathrm{GHz}$.

\begin{tabular}{cccc}
\hline \multicolumn{2}{c}{$\begin{array}{c}\text { Calculated Value } \\
(\mathrm{dB} / \mathrm{km})\end{array}$} & $\begin{array}{c}\text { Measured } \\
\text { Value }(\mathrm{dB} / \mathrm{km})\end{array}$ \\
\hline $\boldsymbol{A}_{\boldsymbol{c}}$ & $\boldsymbol{A}_{\boldsymbol{c}}$ & $\boldsymbol{A}_{\boldsymbol{m}}$ & $\begin{array}{c}\text { Visibility } \\
(\mathrm{km})\end{array}$ \\
$\begin{array}{c}\text { (Poldhirsh } \\
\text { Model) }\end{array}$ & $\begin{array}{c}\text { Model) } \\
0.02\end{array}$ & & \\
0.01 & 0.13 & 0.14 & 0.625 \\
0.007 & 0.064 & 0.1 & 1.25 \\
0.003 & 0.06 & 0.071 & 1.42 \\
\hline 0.002 & 0.021 & 0.05 & 3.75 \\
\hline
\end{tabular}

The signal attenuation $(\mathrm{dB} / \mathrm{km})$ versus different values of visibility plot at frequency equal to $40 \mathrm{GHz}$ for measured and calculated values is shown in Fig. 9. Unlike the predicted values from Goldhirsh model the attenuation values calculated by the proposed model show close agreement to measurements. 


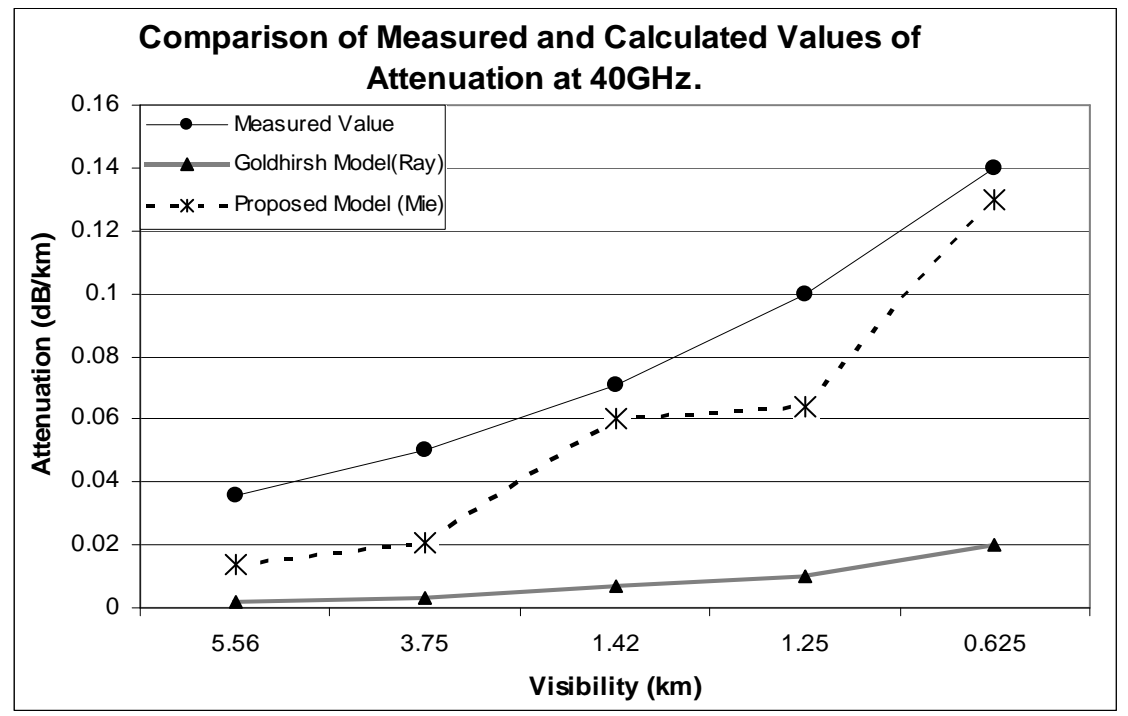

Fig. 9: Comparison between Measured and Calculated Attenuation.

\section{CONCLUSION}

A model has been developed to predict microwave attenuation due to dust particles using Mie solution of Maxwell's equations for the scattering of electromagnetic wave. In this proposed model the term visibility $(V)$ is applied to denote the degree of duststorm density instead of total number of dust particles $(N)$.

The predictions show that the attenuation varies from $13 \mathrm{~dB} / \mathrm{km}$ to $0.2 \mathrm{~dB} / \mathrm{km}$ at 40 $\mathrm{GHz}$ for dust particle radius equal to $50 \mu \mathrm{m}$ and the visibility varies from $10 \mathrm{~m}$ to $500 \mathrm{~m}$. At higher frequency of $100 \mathrm{GHz}$, the attenuation varies from $47 \mathrm{~dB} / \mathrm{km}$ to $2 \mathrm{~dB} / \mathrm{km}$ for dust particle radius equal to $50 \mu \mathrm{m}$ and the visibility varies from $10 \mathrm{~m}$ to $500 \mathrm{~m}$.

The predicted values from the mathematical model are compared with the measured values observed in Saudi Arabia and Sudan. The proposed model predicts the measured data more accurately than existing model. The accurate prediction is useful to design higher frequency links at areas affected by duststorms.

\section{REFERENCES}

[1] R. K. Crane, Propagation Phenomena Affecting Satellite Communication Systems Operating in the Centimeter and Millimeter Wavelength Bands, Proc. IEEE, 59(2), 173-88, 1971.

[2] K. Williams and R. Greeley, Radar Attenuation by Sand: Laboratory Measurements of Radar Transmission, IEEE Transactions on Geoscience and Remote Sensing, vol. 39, No. 11, Nov 2001.

[3] G. Brussaard and P.A. Watson, Atmospheric Modelling and Millimeter Wave Propagation (Ghapman \& Hall, UK 1995). 
[4] Ishimaru, Wave Propagation and Scattering in random media (IEEE, N.Y. 1997).

[5] B.R. Vishvakarma and C.S. Rai, Limitations of Rayleigh Scattering in The Prediction of Millimeter Wave Attenuation in Sand and Dust Storms, Geoscience and Remote Sensing Symposium, IEEE Intr., 1993.

[6] Collin R., E., Antenna \& radiowave propagation (McGraw-hill International Edition, Singapore, 1985).

[7] V. E. Cachorro, New Improvements For Mie Scattering Calculations, eprintarXiv:physics/0103052, March 2001.

[8] S. I. Ghobrial and S. M. Sharief, Microwave attenuation and cross polarization in dust storms, IEEE Trans. Antennas Propagat., v. AP-35, pp. 418-425, Apr. 1987.

[9] T. S. Chu, "Effects of sandstorms on microwave propagation." Bell Sys. Tech. J.. v. 58, no. 2, Feb. 1979.

[10] J. Goldhirsh, A parametric review and assessment of attenuation and backscatter properties associated with dust storms over desert regions in the frequency range of 1 to $10 \mathrm{GHz}$, IEEE Trans. Antennas Propagation, vol. AP-30, no. 6, pp. 1121-1127, 1982.

[11] R. R. Rogers, Statistical Rainstorm Models: Their Theoretical and Physical Foundations, IEEE Trans. On Antennas and Propagation, July 1976.

[12] R. L. Olsen et al., The $a R^{b}$ relation in the calculation of rain attenuation, IEEE Trans. On Antennas and Propagation, vol. AP-26, no. 2, Mar. 1978.

[13] Oguchi, Electromagnetic Wave propagation and Scattering in hydrometeors, Proceedings of The IEEE, vol.71,No.9, Sep 1983.

[14] J. Goldhirsh, "Attenuation and Backscatter from a Derived Two-Dimensional Duststorm Model" IEEE Trans. Antennas Propagation, v. 49, no. 12, pp. 1703-1711, 2001.

[15] S. I. Ghobrial, The effect of sand storms on microwave propagation, Proc. Nat. Telecommun. Conf., Houston, TX. vol. 2, Proc. No. CH1539-6/80/0000-0216, pp. 43.5.1-43.5.4, 1980.

[16] Y. Ruike, W. Zhensen, and Y. Jinguang, The Study Of MMW and MW Attenuation Considering Multiple Scattering Effect in Sand and Dust Storms at Slant Paths, International Journal of Infrared and Millimeter Waves, Vol. 24, No. 8, Aug 2003.

[17] Alhaider, M.A. Ali, A.A., Experimental studies on Millimeterwave and Infrared propagation in Arid Land: The Effect of Sand Storms, Sixth International Conference on Antennas and Propagation ICAP, 1989.

[18] Zain Elabdin Omer Elshaikh, Md. Rafiqul Islam, Othman O. Khalifa and Momoh Jimoh E Salami, Duststorm Measurements for the Prediction of Attenuation on Microwave Signals in Sudan, Submitted to International Conference on Computer and Communication Engineering 2008 (ICCCE'08) Kuala Lumpur, May 2008. 